on as rare exceptions. The experience of this war has shown the phenomenon to be by no means uncommon, so that a few months after an injury even the worst form of electrical response is not in itself of bad prognostic import. We often find that a muscle which has recovered some voluntary power, but not faradic reaction, nevertheless shows definite improvement when tested with the condenser instrument; but this is by no means always the case. Nevertheless, we may always be sure when we find that high condenser numbers are still required (old galvanic response), that at some time in the past there was complete bleckage of all motor impulses, indicating a severe injury to a motor nerve. These points have been insisted on by the present writer in various papers, and failure to appreciate them is responsible for much of the criticism which has been levelled against electro-diagnosis and prognosis. It will thus be seen that the so-called "reaction of degeneration" may occur in a muscle which has clinically recovered, and it would therefore be better to drop the term altogether, and to state simply the length of condenser impulse required to excite a muscle at a given voltage.

As to Captain Milner's argument concerning the electrical conducting powers of the medullary sheath, I am at a loss to follow it. A muscle will still respond to faradism (on short condenser impulses) when the nerve-endings are thrown out of action by curare; therefore, as is pointed out by Lewis Jones in his text-book of "Medical Electricity," the failure of response must be due to some change within the muscle, and cannot be dependent upon the condition of the sheath of the motor nerve. Whatever this change may be, it can obviously persist after voluntary power is re-established ; and in this connexion it is interesting to note that voluntary movement is at first usually sluggish, and the contraction resembles that of unstriped muscle. There would appear to be scope here for pathological investigation.

The whole subject of electro-diagnosis and prognosis bristles with difficulties, and the path of the beginner is beset with gins and pitfalls. Nevertheless, it is a most fascinating branch of medicine, enabling those versed in it to prophesy with confidence and success. Any of your readers interested in the matter will find it discussed in detail in a paper which I read before the Section of Surgery, Royal Society of Medicine, and which was published in THE LANCET of Feb. 19th, 1916.

I am, Sir, yours faithfully, Francis Hernaman.Johnson.

Cavendish-square, W., June 3rd, 1916.

\section{ON THE ILL-TREATMENT OF GENITAL PROLAPSE.}

To the Editor of THE LANCET.

SrR,-Dr. W. E. Fothergill gibes at ventrifixation and hysterectomy as operative measures in prolapse, and yet recommends as part of a curative procedure an equally futile measure-repair of the perineum. It takes no genius to discover the value of the perineum or the so-called perineal body in supporting the pelvic viscera. All one has to do is to pass one finger into the rectum and the thumb into the vagina, and to feel what is in the tissues between. To begin with, the so-called perineal body which is grasped in this way, and which in anatomical sections appears, and in diagrams is represented as, a wedge-shaped mass of tissue of some size, can be flattened quite easily from before backwards into a thin septum; and the only important structure in it is the ring sphincter of the anus, which can easily be felt. In the next place, the perineal body is found not infrequently completely ruptured, without any sign or symptom of prolapse, even when the tear was caused years ago and the patient has been living an active life. How rarely are patients who come for treatment for incontinence of fæces from this cause found to be suffering from prolapse? I have never seen a single one. Unfortunately, Dr. Fothergill appears ignorant of these facts or of their meaning; and thus it is not to be expected he can deduce from them the conclusion that repair of the perineum is of no value in the treatment of prolapse.

As regards the other measures recommended by Dr. Fothergill, experience has shown they are equally unreliable. He says "all can be set right" by plastic vaginal surgery. $\mathrm{He}$ makes no reference to the percentage of recurrences after such operations in his cases; his words suggest he has no recurrences. If so, he must indeed be happy; and he attributes none of his success to the invaluable concomitant of plastic vaginal surgery-rest in bed. Rest in bed, by itself, will cure some cases of prolapse; it will even cure them after the bases of the broad ligaments have been injected with quinine. Rest in bed and an easy time subsequently will work wonders - far greater wonders than any plastic vaginal surgery.

The reason the operative procedure in many cases of prolapse is so bad, and is so often followed by recurrence, is that the nature of the condition is not yet generally understood. Prolapse is of the nature of a hernia; it is a hernia of pelvic viscera through an opening in the muscular parietes, closing in the bony pelvic outlet (an outlet measuring roughly 4 in. by 5 in.), just as a protrusion in the inguinal, femoral, or umbilical regions of abdominal contents through the muscular parietes of the abdomen is a hernia. The content of the protrusion is of secondary importance ; in prolapse the protrusion may consist of bladder, uterus or rectum, which as they come down invaginate the vagina, these various parts participating in different cases in different degrees according to their arrangement and the direction of the displacing force, just as an inguinal hernia may contain intestine, or omentum, or the gall-bladder or the ovary. And each condition arises in the same way : both are produced by strain -frequently repeated. In prolapse, strain causes whatever viscus is over the natural aperture in the pelvic parietes to "come down" through the natural aperture in the parietes, just as strain causes whatever viscus is against the natural aperture in the abdominal parietes to protrude. The protrusion occurs because the natural aperture is larger than normal, or because the striated muscle bounding it has become weaker, and so the aperture can be more easily dilated than normal. Prolapse often follows the resumption of active life soon after childbirth, because childbirth often causes extensive injury of the pelvic muscular parietes, and leads to permanent enlargement of the natural aperture in that parietes; but prolapse often appears for the first time years after childbirth, because the muscular parietes have not been sufficiently injured at childbirth and have remained strong enough for their work and only subsequently with advancing years have become weak.

Surgeons do not operate on hernia in people with weakened abdominal walls, because in such cases the hernia tends to recur, and often does recur, and success is unlikely; yet gynæcologists expect to cure prolapse in all cases. With too few exceptions they pay no attention to the muscular parietes of the pelvis, which, according to Dr. Fothergill, are vestigial in structure and tail-moving in origin. In the cases referred to surgeons advise a truss; they do not recommend a plastic operation on the skin and subcutaneous tissues covering the hernial protrusion or placed around it, or advocate plastic operations ou the attachments of the viscera inside the abdomen, comparable to the plastic vaginal surgery performed for prolapse. They do not, for instance, shorten mesenteries - although in hernia the mesenteries have become longer. Or, if they operate, they insert a large filigree over the hernial site ; they do not, however, place this outside the muscular wall in the fat beneath the skin. They place it inside the muscular wall, between it and the peritoneum.

It is left for gynæcologists to recommend a plastic operation on the skin and superficial fascia, and on the viscera themselves inside the pelvis, to overcome a similar complaint. Dr. Fothergill may "repair the perineum," perform colporrbaphy, and with the same breath decry the use of pessaries. But pessaries are but filigrees in a different form, and they are placed in the same relative position as that used by McGavin in his abdominal operations. Truly, they are placed within the vagina, but they are placed in that part of the vagina which is within the pelvic cavity, which is above the pelvic floor-the pelvic muscular parietes-between the pelvic floor and superjacent visceral structures. The use of the pessary in many cases of prolapse is the finest, the best, and often the only efficacious treatment. This is not only the experience of ages and of present-day practitioners of medicine, including many real specialists; it is also the result of a searching theoretical and academic inquiry which will bear probing even to its foundations.

I am, Sir, yours faithfully,

Rugby, June 5th, 1916. R. H. PARAMORE, F.R.C.S. Eng. 